\title{
Erratum
}

\section{The Third French Individual and National Food Consumption (INCA3) Survey 2014-2015: method, design and participation rate in the framework of a European harmonization process - ERRATUM}

\author{
Carine Dubuisson, Ariane Dufour, Sandrine Carrillo, Peggy Drouillet-Pinard, \\ Sabrina Havard and Jean-Luc Volatier
}

First published online 27 December 2018

doi: 10.1017/S1368980018002896, Published online by Cambridge University Press, 5 November 2018

The above article was published without standard deviations for 'Number of days between first and last R24' in Table 5 . Values for children are $\mathrm{SD}=20.2$ and for adults $\mathrm{SD}=28.6$.

\section{Original text}

Table 5 Description of the dietary interviews in the INCA3 survey according to the sample (row data)

\begin{tabular}{|c|c|c|c|c|}
\hline & \multicolumn{2}{|c|}{ Children } & \multicolumn{2}{|c|}{ Adults } \\
\hline Information at individual level & \multicolumn{2}{|c|}{ (n 1993) } & \multicolumn{2}{|c|}{ (n 2121) } \\
\hline Only two R24, \% & \multicolumn{2}{|c|}{2.6} & \multicolumn{2}{|c|}{3.8} \\
\hline Only weekdays, \% & \multicolumn{2}{|c|}{$1 \cdot 2$} & \multicolumn{2}{|c|}{2.6} \\
\hline Only weekend days, \% & \multicolumn{2}{|c|}{0.3} & \multicolumn{2}{|c|}{0.4} \\
\hline Number of days between first and last R24, mean and SD & \multirow{2}{*}{\multicolumn{2}{|c|}{$\begin{array}{c}21.7 \\
(n 5927)\end{array}$}} & \multirow{2}{*}{\multicolumn{2}{|c|}{$\begin{array}{c}36 \cdot 3 \\
(n 6283)\end{array}$}} \\
\hline Information at day level & & & & \\
\hline Interview duration (min), mean and SD & $30 \cdot 8$ & 11.9 & $39 \cdot 4$ & $16 \cdot 6$ \\
\hline \multicolumn{5}{|l|}{ Number of reported foods/d, mean and SD } \\
\hline Day 1 & $18 \cdot 8$ & $5 \cdot 3$ & $24 \cdot 3$ & 7.5 \\
\hline Day 2 & $18 \cdot 0$ & $5 \cdot 6$ & 23.7 & $7 \cdot 3$ \\
\hline Day 3 & $17 \cdot 4$ & 5.5 & 23.3 & 7.5 \\
\hline Total & $18 \cdot 0$ & 5.5 & $23 \cdot 8$ & 7.5 \\
\hline \multicolumn{5}{|l|}{ Individual energy intake $(\mathrm{kcal}) \dagger$, mean and SD } \\
\hline Day 1 & 1805 & 726 & 2092 & 875 \\
\hline Day 2 & 1757 & 693 & 2067 & 880 \\
\hline Day 3 & 1724 & 707 & 2039 & 847 \\
\hline Total & 1763 & 709 & 2067 & 868 \\
\hline \multicolumn{5}{|l|}{ Day repartition, \% } \\
\hline Monday & \multicolumn{2}{|c|}{$14 \cdot 9$} & \multicolumn{2}{|c|}{$14 \cdot 3$} \\
\hline Tuesday & \multirow{2}{*}{\multicolumn{2}{|c|}{$\begin{array}{l}14.7 \\
11.9\end{array}$}} & \multicolumn{2}{|c|}{$18 \cdot 8$} \\
\hline Wednesday & \multirow{2}{*}{\multicolumn{2}{|c|}{$\begin{array}{l}11.9 \\
13.7\end{array}$}} & \multicolumn{2}{|c|}{$11 \cdot 2$} \\
\hline Thursday & & & \multicolumn{2}{|c|}{$14 \cdot 8$} \\
\hline Friday & \multicolumn{2}{|c|}{11.4} & \multicolumn{2}{|c|}{$7 \cdot 7$} \\
\hline Saturday & \multicolumn{2}{|c|}{$17 \cdot 1$} & \multicolumn{2}{|c|}{$13 \cdot 2$} \\
\hline Sunday & \multicolumn{2}{|c|}{$16 \cdot 3$} & & \\
\hline \multicolumn{5}{|l|}{ Season repartition, \% } \\
\hline Winter & \multicolumn{2}{|c|}{33.4} & \multicolumn{2}{|c|}{$25 \cdot 6$} \\
\hline Spring & \multicolumn{2}{|c|}{$29 \cdot 9$} & & \\
\hline Summer & & & & \\
\hline Autumn & & & & \\
\hline
\end{tabular}

INCA3, Third French Individual and National Food Consumption Survey 2014-2015; R24, $24 \mathrm{~h}$ recall/record.

†To convert to kJ, multiply kcal values by $4 \cdot 184$. 


\section{Correction}

Table 5 Description of the dietary interviews in the INCA3 survey according to the sample (row data)

\begin{tabular}{|c|c|c|c|c|c|}
\hline & & Children & & Adults & \\
\hline Information at individual level & & (n 1993) & & (n 2121) & \\
\hline Only two R24, \% & & 2.6 & & 3.8 & \\
\hline Only weekdays, \% & & $1 \cdot 2$ & & $2 \cdot 6$ & \\
\hline Only weekend days, $\%$ & & 0.3 & & 0.4 & \\
\hline Number of days between first and last R24, mean and SD & $21 \cdot 7$ & $20 \cdot 2$ & $36 \cdot 3$ & & $28 \cdot 6$ \\
\hline Information at day level & & (n 5927) & & $(n 6283)$ & \\
\hline Interview duration (min), mean and SD & $30 \cdot 8$ & 11.9 & 39.4 & & $16 \cdot 6$ \\
\hline \multicolumn{6}{|l|}{ Number of reported foods/d, mean and SD } \\
\hline Day 1 & $18 \cdot 8$ & $5 \cdot 3$ & $24 \cdot 3$ & & 7.5 \\
\hline Day 2 & $18 \cdot 0$ & 5.6 & 23.7 & & $7 \cdot 3$ \\
\hline Day 3 & $17 \cdot 4$ & 5.5 & $23 \cdot 3$ & & 7.5 \\
\hline Total & $18 \cdot 0$ & 5.5 & $23 \cdot 8$ & & 7.5 \\
\hline \multicolumn{6}{|l|}{ Individual energy intake $(\mathrm{kcal}) \dagger$, mean and SD } \\
\hline Day 1 & 1805 & 726 & 2092 & & 875 \\
\hline Day 2 & 1757 & 693 & 2067 & & 880 \\
\hline Day 3 & 1724 & 707 & 2039 & & 847 \\
\hline Total & 1763 & 709 & 2067 & & 868 \\
\hline \multicolumn{6}{|l|}{ Day repartition, \% } \\
\hline Monday & & 14.9 & & $14 \cdot 3$ & \\
\hline Tuesday & & $14 \cdot 7$ & & $18 \cdot 8$ & \\
\hline Wednesday & & 11.9 & & $11 \cdot 2$ & \\
\hline Thursday & & 13.7 & & 14.8 & \\
\hline Friday & & 11.4 & & $7 \cdot 7$ & \\
\hline Saturday & & $17 \cdot 1$ & & $13 \cdot 2$ & \\
\hline Sunday & & $16 \cdot 3$ & & $20 \cdot 0$ & \\
\hline \multicolumn{6}{|l|}{ Season repartition, \% } \\
\hline Winter & & 33.4 & & $25 \cdot 6$ & \\
\hline Spring & & 29.9 & & $36 \cdot 0$ & \\
\hline Summer & & $19 \cdot 5$ & & $22 \cdot 6$ & \\
\hline Autumn & & $17 \cdot 2$ & & $15 \cdot 8$ & \\
\hline
\end{tabular}

INCA3, Third French Individual and National Food Consumption Survey 2014-2015; R24, $24 \mathrm{~h}$ recall/record. †To convert to $\mathrm{kJ}$, multiply kcal values by $4 \cdot 184$.

\section{Reference}

Dubuisson C, Dufour A, Carrillo S, Drouillet-Pinard P, Havard S, \& Volatier J (n.d.) The Third French Individual and National Food Consumption (INCA3) Survey 2014-2015: Method, design and participation rate in the framework of a European harmonization process. Public Health Nutrition, 1-17. doi:10.1017/\$1368980018002896 